\title{
Presença de formas amastigotas de Leishmania chagasi e perfil leucocitário no aparelho reprodutivo de cães ${ }^{1}$
}

\author{
Ariane P. Benites ${ }^{2}$, Carlos E. Fernandes ${ }^{3}$, Karine B. Brum ${ }^{3}$ \\ e Maria Auxiliadora G.S. Abdo ${ }^{3}$
}

\begin{abstract}
Benites A.P., Fernandes C.E., Brum K.B. \& Abdo M.A.G.S. 2011. [Presence of amastigotes forms the Leishmania chagasi and profile the leucocytes cells in the reproductive tract of dogs.] Presença de formas amastigotas de Leishmania chagasie perfil leucocitário no aparelho reprodutivo de cães. Pesquisa Veterinária Brasileira 31(1):7277. Faculdade de Medicina Veterinária e Zootecnia, Universidade Federal de Mato Grosso do Sul, Cidade Universitária, Caixa Postal 549, Campo Grande, MS 79070-900, Brazil. E-mail: arianebenites@ hotmail.com

Visceral leishmaniasis (VL) is a zoonosis caused by Leishmania (Leishmania) chagasi. Canine visceral leishmaniasis (VLC) is most important. The infection occurs usually between the invertebrate host and vertebrate host; however, transmission in the absence of the vector has been reported. The aim of this study was to identify the presence of amastigote forms, quantify the leucocyte cells and to estimate the presence (odds ratio) of the amastigotes in the reproductive tract of dogs serologically positive with and without clinical signs. Sexually mature Mongrel dogs, serologically tested to VLC (symptomatic, $n=25$; asymptomatic, $n=25$ ), were used. After euthanasia, testes, epidydimal (caput, corpus and cauda) and prostate gland fragments (randomized) were recovered and impressed on slides. Twenty animals serologically negative and asymptomatic were used as control group. Samples of spleen were included as parasitological positive controls. Lymphocyte percentages were higher $(\mathrm{P}<0.05)$ in the corpus and caudal region of epididymis, similar to the testes in the symptomatic group. Macrophage percentage was higher $(P<0.05)$ in the corpus and caudal epididymis regions. The presence of amastigote forms was associated with different regions of the reproductive tract. In the symptomatic group, the variation was between 0.50 and 0.80 , and in the symptomatic between 0.79 and 0.95 . The odds ratio for amastigote forms in the testicle of the symptomatic dogs was 6.5 in relation to asymptomatic dogs. The results demonstrate the epidemic potential of venereal transmission of the disease, specifically in areas where control programs of VLC do not consider this transmission route.
\end{abstract}

INDEX TERMS: Visceral leishmaniasis, Leishmania, leucocytes, epidydimis, testis, dogs.

RESUMO.- A leishmaniose visceral (LV) é uma zoonose causada pelo protozoário Leishmania (Leishmania) chagasi. A leishmaniose visceral canina (LVC) é a doença de maior

\footnotetext{
1 Recebido em 16 de abril de 2010.

Aceito para publicação em 20 de setembro de 2010.

Parte da Dissertação de Mestrado em Ciência Animal, Faculdade de Medicina Veterinária e Zootecnia (FAMES), Universidade Federal de Mato Grosso de Sul, Campo Grande, MS 79070-900.

2 Programa de Pós-Graduação em Ciência Animal, FAMES/UFMS, Cidade Universitária, Cx. Postal 549, Campo Grande, MS 79070-900. *Autor para correspondência: arianebenites@ hotmail.com

${ }^{3}$ Departamento de Patologia, Centro de Ciências Biológicas e de Saúde, CCBS, UFMS, Cidade Universitária, Cx. Postal 549, Campo Grande, MS 79070-900.
}

relevância zoonótica. Usualmente, a infecção ocorre entre um hospedeiro invertebrado para um hospedeiro vertebrado, entretanto, a transmissão na ausência do vetor já é conhecida. O objetivo principal deste estudo foi identificar a presença de formas amastigotas, quantificar as células leucocitárias, estimar o risco relativo da presença de formas amastigotas no aparelho reprodutivo de cães sorologicamente positivos com e sem sinais clínicos. Para isso, foram utilizados cães sem raça definida, sexualmente maduros e testados sorologicamente para LVC (com sinais clínicos, $n=25$; sem sinais clínicos, $n=25$ ), que após eutanásia, tiveram fragmentos de testículo, epidídimo (cabeça, corpo e cauda) e glândula prostática (selecionados 
ao acaso) impressos em lâminas. Um grupo de 20 cãs sorologicamente negativos e sem sinais clínicos foi usado como controle. Amostras do baço foram incluídas como controle parasitológico positivo. O percentual de linfócitos foi superior $(\mathrm{P}<0,05)$ no corpo e cauda do epidídimo, assim como no testículo. Macrófagos foram superiores $(P<0,05)$ apenas nas regiões do corpo e cauda epididimais. A presença de amastigotas correlacionou-se entre as distintas regiões do aparelho reprodutivo. Nos sintomáticos variaram entre 0,50 a 0,80 e entre 0,79 a 0,95 nos assintomáticos. A presença de amastigotas no testículo dos cães sintomáticos foi 6,5 vezes superior aos cães assintomáticos. Os resultados obtidos demonstram o potencial epidemiológico da transmissão venérea da doença, principalmente em áreas onde os programas de controle da LVC não consideram esta forma de transmissão, que pode ser importante em populações caninas não esterilizadas.

TERMOS DE INDEXAÇÃO: Leishmaniose visceral canina, Leishmania, leucócitos, epidídimo, testículo.

\section{INTRODUÇÃO}

A leishmaniose visceral (LV) é uma zoonose causada pela Leishmania (Leishmania) chagasi (Deane \& Deane 1962). A leishmaniose visceral canina (LVC) é a doença de maior relevância zoonótica causada por um protozoário que afeta humanos e cães na Europa e América Latina (Desjeux 2004) e um sério problema de saúde pública (Tesh 1995, França-Silva et al. 2003). Essa doença é endêmica em 88 países e aproximadamente $90 \%$ dos casos mundialmente notificados ocorrem em Bangladesh, Brasil, Índia, Nepal e Sudão (WHO 2003). O Brasil é responsável por $90 \%$ dos casos de LV no continente americano (Monteiro et al. 1994).

A LVC pode apresentar-se desde uma forma assintomática até uma doença sistêmica, muitas vezes levando o cão à morte. A partir da infecção cutânea localizada, o parasita pode se disseminar pela via linfática ou pelos vasos sanguíneos, infectando macrófagos da medula óssea, linfonodos, fígado e baço, assim como nos rins e trato gastrointestinal (Tryphomas et al. 1977, Keenan et al. 1984). Inicialmente, os sinais clínicos são linfadenomegalia superficial generalizada, edema de membros e dermatite esfoliativa, inicialmente periorbital e nasal, e posteriormente disseminada, caracterizada por alopecia, descamação e onicogrifose. Outros sinais como febre, apatia, diarreia, emagrecimento progressivo, hepatomegalia, esplenomegalia, hiperceratose, ulcerações cutâneas, particularmente no focinho e orelha, e ceratoconjuntivite são frequentes, apesar de não estarem presentes em todos os casos (Genaro et al. 1988, Dias et al. 1999). De acordo com os sinais clínicos, Mancianti et al. 1988 classificou os cães infectados em: sintomáticos, aqueles que apresentam mais de três sinais clínicos; oligossintomáticos, de um a três sinais clínicos e assintomáticos sem nenhum sinal clínico.

O cão é considerado o reservatório mais importante da LV (Moreno \& Alvar 2002). A transmissão do agente é feita por várias espécies de flebotomíneos. No Brasil, os insetos responsáveis pela transmissão são do gênero Lutzomyia (Galati et al. 1997). Usualmente, a infecção ocorre entre um hospedeiro invertebrado para um hospedeiro vertebrado, entretanto, a transmissão na ausência do vetor já é conhecida (Marsella \& Gopegui 1998). Um caso de transmissão direta através de transfusão sanguínea já foi documentado em um cão (Owens et al. 2001). Recentemente, Silva et al. (2009) comprovaram que a Leishmania chagasi pode ser sexualmente transmissível de cães machos naturalmente infectados para fêmeas susceptíveis na ausência do vetor biológico. No entanto, embora haja essa perspectiva no cão, poucos estudos têm evidenciado aspectos ligados à patogenia e manifestação clínica da LVC a partir da frequência das formas amastigotas e resposta leucocitária no aparelho reprodutivo e fluídos seminais.

O objetivo deste estudo foi identificar a presença de formas amastigotas, quantificar as células leucocitárias e estimar o risco relativo da presença de formas amastigotas no aparelho reprodutivo de cães com LVC com ou sem sinais clínicos.

\section{MATERIAL E MÉTODOS}

\section{Animais}

Foram utilizados 70 cães machos sexualmente maduros, sem raça definida, preliminarmente testados por meio de exame sorológico para detecção de anticorpos anti-Leishmania, através do ensaio de imunoadsorção enzimática $(E L I S A)^{4}$ e da reação de imunofluorescência indireta $(\mathrm{RIFI}, 1: 40)^{5}$ provenientes do Centro de Controle de Zoonoses do município de Campo Grande, MS (20 262343 S, 54 382453 W).

Os cães foram divididos em três grupos, de acordo com a classificação de Mancianti et al. (1988): Grupo $1(n=25)$ sorologicamente positivos, sintomáticos; Grupo $2(n=25)$ sorologicamente positivos, assintomáticos e Grupo $3(n=20)$ sorologicamente negativos/controle, sem sinais clínicos. Este experimento foi aprovado pelo Comitê de Ética para uso de animais da Universidade Federal de Mato Grosso do Sul (Protocolo 203/2009).

\section{Exame citológico}

Após eutanásia, foram colhidos fragmentos de testículo, epidídimo (cabeça, corpo e cauda), próstata e baço para realização de impressões em lâmina. As lâminas foram fixadas em metanol e coradas pelo método Panótico (ciclohexadieno 0,1\%, azobenzenosulfônicos $0,1 \%$ e fenotiazina $0,1 \%)^{6}$ e avaliadas em microscópio de campo claro sob 40x. As impressões citológicas foram usadas para estimar a frequência, tipo de leucócitos e células de descamação nas diferentes regiões do aparelho reprodutivo, mediante leitura de 20 campos/lâmina escolhidos ao acaso. A presença ou a ausência de amastigotas foi determinada em microscopia de campo claro sob imersão (100x) nos diferentes segmentos do aparelho reprodutivo. As amostras do baço foram utilizadas como controle parasitológico positivo.

\footnotetext{
${ }^{4}$ Bio-Manguinhos - Fundação Oswaldo Cruz, Av. Brasil 4365, Rio de Janeiro, RJ.

${ }^{5}$ Bio-Manguinhos - Fundação Oswaldo Cruz, Av. Brasil 4365, Rio de Janeiro, RJ.

${ }^{6}$ Panótico Rápido LB®, Laborclin Produtos para Laboratórios Ltda, Rua Cassemiro de Abreu 521, Pinhais, PR.
} 


\section{Análise estatística}

A frequência das formas amastigotas entre os grupos e nos diferentes segmentos avaliados foi comparada pelo teste do Qui-quadrado (tabelas de contingência, $P<0,05$ ). A comparação do perfil leucocitário e de células de descamação entre regiões do aparelho reprodutivo e grupos experimentais foi realizada pela análise de variância e o modelo linear multivariado (teste de Duncan, $\mathrm{P}<0,05$ ), considerando os efeitos principais e interação (regiões $x$ grupos). A associação da presença das formas amastigotas entre regiões do aparelho reprodutivo e baço foi estimada pela análise da correlação (modelo binário proximidade entre variáveis, método simple matching). A taxa de risco (odds ratio) para o aparecimento de amastigotas em diferentes segmentos do aparelho reprodutivo entre sintomáticos e assintomáticos foi estimada pela análise de regressão logística múltipla (Ayres et al. 2005).

\section{RESULTADOS}

A presença de formas amastigotas de Leishmania foi identificada nas diferentes regiões do aparelho reprodutivo (Fig.1). O percentual da presença ou ausência de formas amastigotas entre os grupos de cães sorologicamente po-

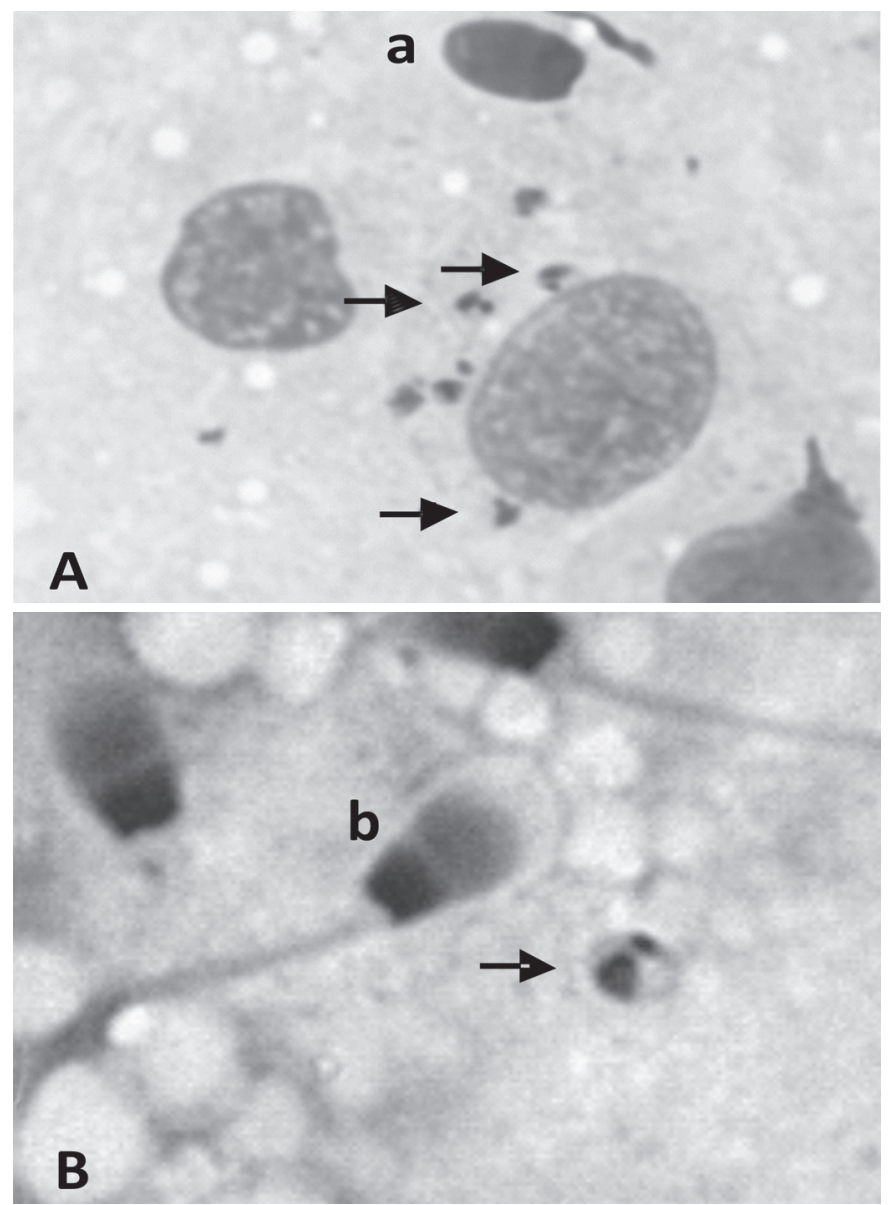

Fig.1. Formas amastigotas de Leishmania chagasi em cães sorologicamente positivos (ELISA e RIFI). (A) Impressão de testículo; (a) cabeça espermática. (B) Impressão de epidídimo; (b) espermatozóide. (setas indicam formas amastigotas). Coloração Panótico, obj.100x.

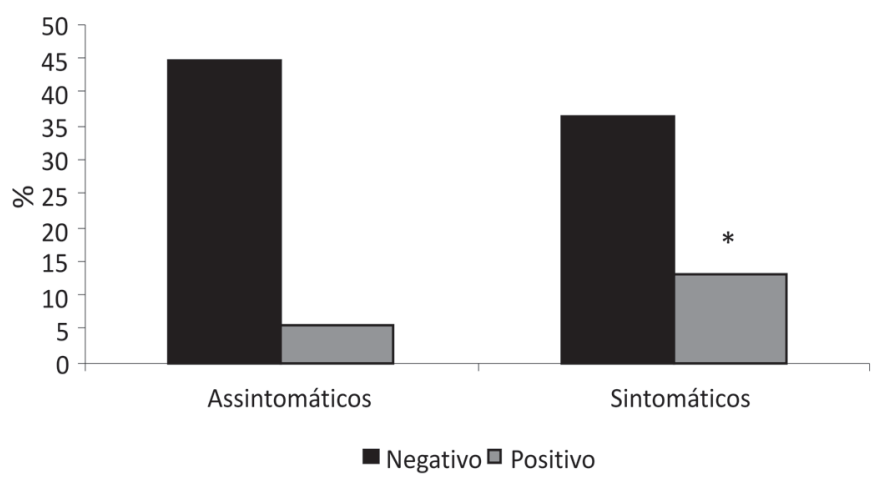

Fig.2. Frequência de formas amastigotas no aparelho reprodutivo de cães sorologicamente positivos para LVC de acordo com manifestação clínica ( ${ }^{*} P<0,05$ entre cães positivos).

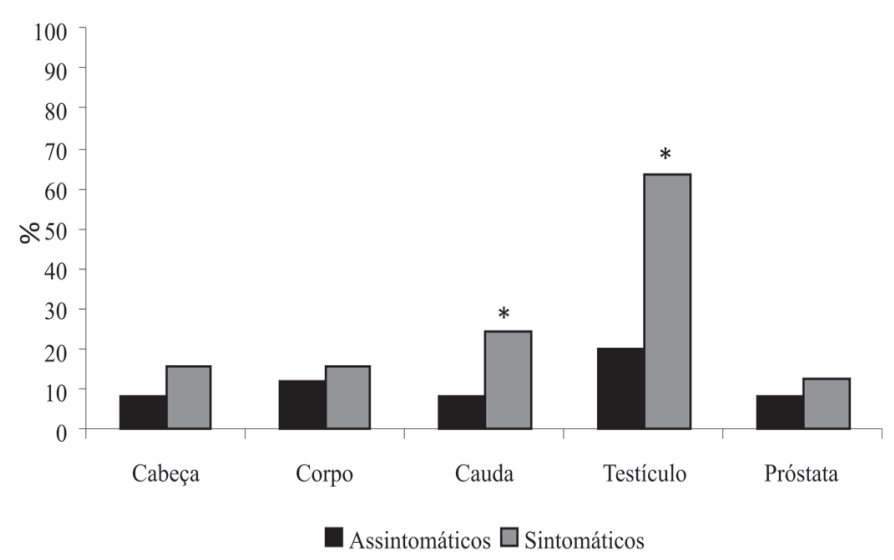

Fig.3. Frequência de formas amastigotas nas diferentes regiões do aparelho reprodutivo de cães sorologicamente positivos para LVC de acordo com a manifestação clínica ( $\left.{ }^{*} P<0,05\right)$.

sitivos com e sem manifestação clínica da LVC e entre as diferentes regiões do aparelho reprodutivo estão representados nas Figuras 2 e 3.

O perfil leucocitário e das células de descamação entre os grupos experimentais e em cada região do aparelho reprodutivo está representado na Figura 4. Houve diferença significativa $(P<0,05)$ entre grupos e entre regiões do aparelho reprodutivo.

A análise de proximidade para a presença de formas amastigotas entre o baço e as diferentes regiões do apareIho reprodutivo dos cães sorologicamente positivos sintomáticos e assintomáticos para LVC, está demonstrada na Figura 5. Para o grupo de cães assintomáticos verificouse valores maiores entre o baço e o aparelho reprodutivo.

A análise de regressão logística múltipla e odds ratio estão demonstrados no Quadro 1 e o modelo foi significativo para todas as regiões estudadas e importantes para a presença de amastigotas nos cães sintomáticos. Porém, o testículo foi o órgão que apresentou a maior taxa de risco $(P<0,05)$ quanto à presença de formas amastigotas nos cães sorologicamente positivos e sintomáticos (odds ratio $=6,51$ ) com probabilidade estimada para a presença de amastigotas de $69,4 \%$. Nas outras regiões estudadas as taxas de risco foram inferiores. 

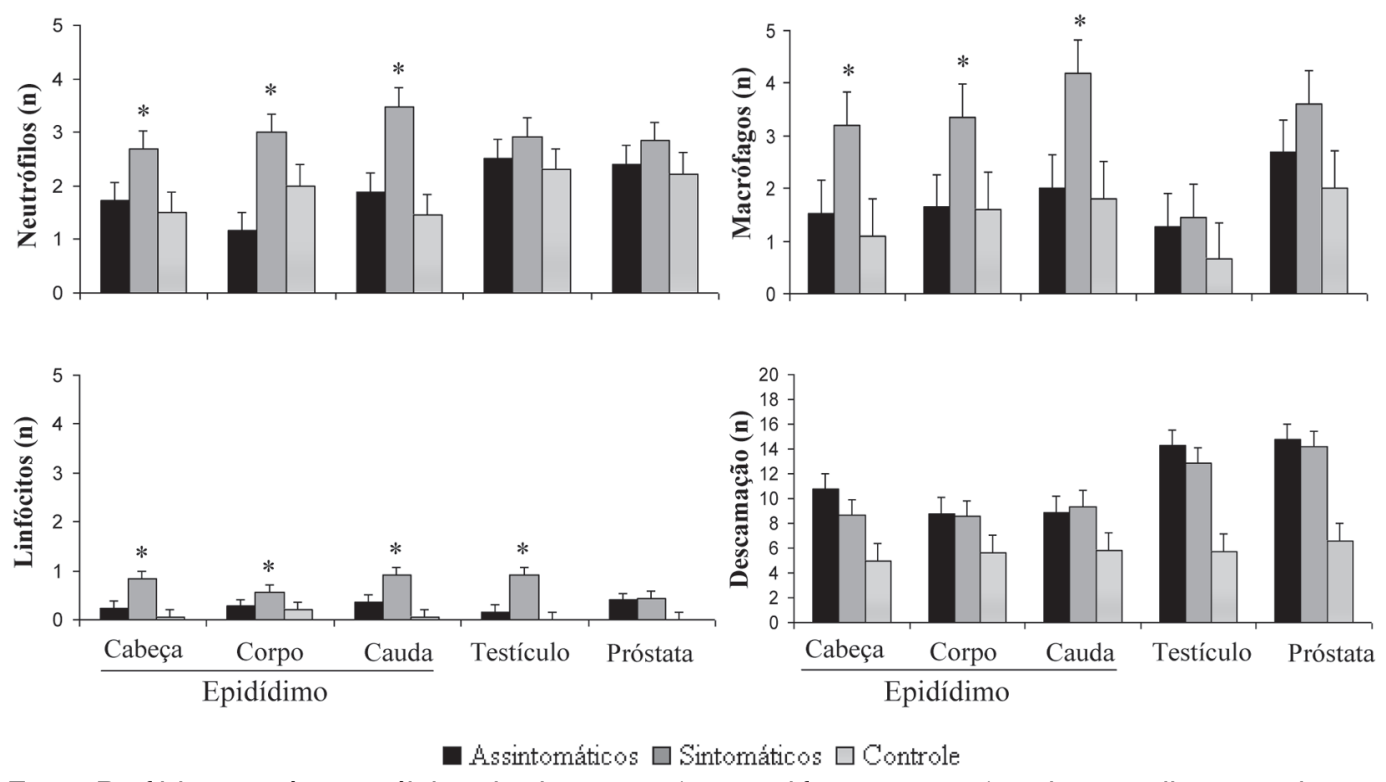

Fig.4. Perfil leucocitário e células de descamação nas diferentes regiões do aparelho reprodutivo de cães sorologicamente positivos para LVC de acordo com manifestação clínica e grupo controle ( $\left.{ }^{*} \mathrm{P}<0,05\right)$.

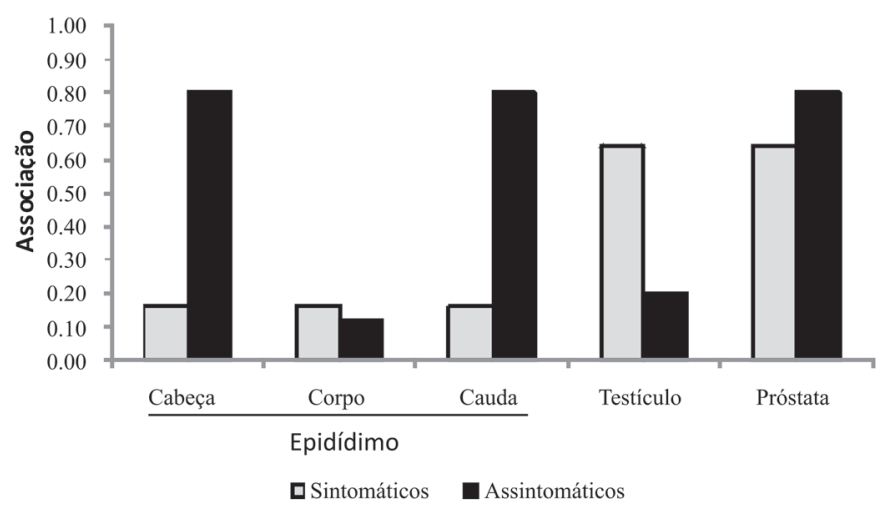

Fig.5. Associação da presença de formas amastigotas entre o baço e as diferentes regiões do aparelho reprodutivo em cães sorologicamente positivos para LVC, de acordo com a manifestação clínica. *Matriz de proximidade estimada pelo método binário, medida simple matching.

\section{DISCUSSÃO}

Os resultados desse estudo demonstraram que o aparelho reprodutivo de cães sorologicamente positivos para LVC pode ser um reservatório para formas infectantes. Na cau- da do epidídimo e nos testículos houve maior prevalência de amastigotas nos cães que apresentavam sinais clínicos da doença, como previamente descrito (Diniz et al. 2005). A manifestação clínica da leishmaniose está na dependência da carga parasitária, considerando o número de formas para cada 1.000 núcleos de células. Isto demonstra, em parte, as diferenças encontradas entre cães com variação na sintomatologia clínica (Reis 2001, Giunchetti et al. 2006, 2008, Reis et al. 2006) e possivelmente nas distintas regiões do aparelho reprodutivo como observado neste estudo.

Paralelamente à presença das amastigotas, verificaram-se alterações no perfil leucocitário com valores maiores para o grupo de cães sintomáticos. Embora não se tenha estimado lesões histopatológicas, os perfis encontrados sugerem que haja, no mínimo, respostas diferenciadas em ambientes diferentes frente às formas amastigotas, caracterizados pelo maior número de neutrófilos, macrófagos e linfócitos. Por outro lado, é possível que tenha havido resposta inflamatória em decorrência da carga parasitária localizada no aparelho reprodutivo. Segundo Diniz et al. (2005), a frequência e a intensidade das lesões infla-

Quadro 1. Estimativa para taxa de risco (odds ratio)* para a presença

de amastigotas de Leishmania chagasi em diferentes regiões do aparelho reprodutivo de cães sorologicamente positivos sintomáticos

\begin{tabular}{ccccccc}
\hline Região & Coeficiente & EP & Z & p-valor & OR & IC 95\% \\
\hline Cabeça do epidídimo & 0,1779 & 1,06 & 0,17 & 0,867 & 1,19 & $0,15-9,53$ \\
Corpo do epidídimo & 0,1471 & 0,98 & 0,15 & 0,8811 & 1,16 & $0,17-7,95$ \\
Cauda do epidídimo & 1,1190 & 0,98 & 1,14 & 0,2551 & 3,06 & $0,45-21,04$ \\
Testículo & 1,8727 & 0,67 & 2,78 & 0,0055 & 6,51 & $1,73-24,40$ \\
Próstata & 0,8177 & 1,08 & 0,76 & 0,4472 & 2,27 & $0,27-18,66$
\end{tabular}

*Análise de regressão logística múltipla

$\left[\right.$ LogitPi $\left.=1,052+\left(0,178 X_{1}\right)+\left(0,147 X_{2}\right)+\left(1,119 X_{3}\right)+\left(1,873 X_{4}\right)+\left(0,818 X_{5}\right)\right] ; \chi^{2}=12,22 ;$ $\mathrm{GL}=5 ; \mathrm{P}=0,0318$. 
matórias no testículo estão correlacionadas com a manifestação clínica da doença, já que o número de amastigotas no testículo aparentemente atua como fator desencadeante da resposta inflamatória. Nos testículos dos cães sintomáticos, houve maior frequência de linfócitos demonstrando uma resposta imune local. A habilidade do testículo em proteger as células germinativas auto-antigênicas do sistema imune tem considerado o testículo como um local imunologicamente privilegiado. A imunotolerância no testículo é amplamente conhecida com base no fato de que as células de Sertoli separam mecanicamente todas as células germinativas (auto-antígenos) por meio da barreira hematotesticular (De Cesaris et al. 1992). O tecido intersticial é rico em macrófagos que expressam $\mathrm{MHC}$ de classe II, linfócitos T e B, presentes na rete testis e ducto eferente, além da grande área ocupada pelos vasos linfáticos e capilares sanguíneos, facilitando a migração de células de defesa (Hedger 1997, Setchell \& Breed 2006). Portanto, a atividade imunocelular do testículo pode contribuir para a susceptibilidade à infecção, explicando dessa forma o alto percentual de cães sorologicamente positivos que apresentaram formas amastigotas neste órgão, associado ao perfil leucocitário diferenciado.

Processos inflamatórios pré-existentes podem recrutar macrófagos contendo Leishmania, como no caso da epididimite (Diniz et al. 2005). Na cabeça, corpo e cauda do epidídimo há notadamente migração de células imunocompetentes, que normalmente situam-se na base do epitélio e se deslocam para o lúmen (Serre \& Robaire 2002). No entanto, fatores que levam ao aumento da prevalência destas células e a resposta imune celular local neste órgão, frente à complexidade da resposta imune sistêmica a LVC não são totalmente conhecidos (Hedger \& Hales 2006). Neste estudo, o perfil leucocitário encontrado nos cães com sintomatologia para LVC sugere maior transmigração de neutrófilos, macrófagos e linfócitos para o lúmen dos segmentos epididimais avaliados (Fig.4). Dessa forma, é possível que a presença de amastigotas esteja de acordo com a maior ocorrência dessas células nestes órgãos do aparelho reprodutivo. Ambos os perfis observados no testículo e epidídimo indicam que nesse grupo de cães há uma resposta imune mais demorada, característica de casos crônicos com alta parasitemia (Reis et al. 2009). Na próstata, o perfil leucocitário não apresentou diferenças significativas entre os grupos avaliados, incluindo o grupo controle. Esses resultados demonstram que não há associação entre o perfil leucocitário e a presença de formas amastigotas neste órgão.

Além dos aspectos associados à frequência e distribuição dos leucócitos nos diferentes órgãos do aparelho reprodutivo, associou-se a presença ou ausência de formas amastigotas com o baço, órgão considerado como controle parasitológico positivo. Essa metodologia permitiu estimar o nível de proximidade a partir da constatação da presença das formas infectantes. A vantagem desse método resumese na não exigência de pressuposição inicial quanto à distribuição de probabilidades dos dados (Everrit 1974). Assim, foi possível ter idéia do grau de dependência dos diferentes segmentos em relação ao baço de acordo com a sintomatologia clínica. O grupo de cães sorologicamente positivos sintomáticos apresentou baixa proximidade entre baço e os órgãos avaliados, exceto para o testículo e próstata. Já no grupo de cães sorologicamente positivos assintomáticos a associação entre baço e demais órgãos avaliados foi alta, principalmente entre cabeça e cauda do epidídimo e próstata (Fig.5). As diferenças encontradas sugerem que há independência entre o parasitismo do baço com o percentual de positividade para a presença de formas amastigotas no aparelho reprodutivo e as variações no perfil leucocitário dessas regiões. Por outro lado, no testículo dos cães sintomáticos, a proximidade moderada com o baço $(0,64)$ demonstra novamente a importância desse órgão na manutenção de formas amastigotas nos casos de LVC. Essa importância é reiterada pela taxa de risco estimada na ordem de 6,5 vezes superior em relação aos cães assintomáticos, com probabilidade de aproximadamente $69 \%$ de ocorrência das formas infectantes no testículo quando há presença de sinais clínicos.

Os resultados deste estudo demonstram que há variações no perfil leucocitário entre cães sintomáticos e assintomáticos para LVC associadas à presença natural de formas amastigotas nos fluidos e nas diferentes regiões do aparelho reprodutivo. Adicionalmente, os testículos podem ser importantes na manutenção de formas infectantes no organismo ou na disseminação para os segmentos extragonadais. Apesar da transmissão pelo vetor biológico ser a mais importante e conhecida rota de transmissão, os resultados obtidos reforçam o potencial epidemiológico da transmissão venérea da doença, principalmente em áreas de ocorrência endêmica da LVC, onde os programas de controle não consideram esta forma de infecção, que podem ter um impacto significante particularmente no Brasil, país em que a maior parte da população canina não é esterilizada.

Agradecimentos.- Ao Centro de Controle de Zoonoses de Campo Grande (MS) e à Pró-Reitoria de Pesquisa e Pós Graduação (PROPP) da Universidade Federal de Mato Grosso do Sul pelo suporte no material experimental.

\section{REFERÊNCIAS}

Ayres M., Ayres Jr M., Ayres D.L. \& dos Santos A.A.S. 2005. Bioestat: aplicações nas áreas das ciências biomédicas. IOEPA, Belém, PA. 324p.

De Cesaris P., Filippini A., Cervelli C., Riccioli A., Muci S., Starace G., Stefanini M. \& Ziparo E. 1992. Immunosuppressive molecules produced by Sertoli cells cultured in vitro: Biological effects on lymphocytes. Biochem. Biophys. Res. Commun. 186:1639-1646.

Deane L.M. \& Deane M.P. 1962. Visceral leishmaniasis in Brazil: Geographical distribution and transmission. Revta Inst. Med. Trop., São Paulo, 4:198.

Desjeux P. 2004. Leishmaniasis: Current situation and new perspectives. Comp. Immunol. Microbiol. Infect. Dis. 27:305-318.

Dias D.V., Da Costa C.A., Toledo V.P.C.P., Bambirra E., Genaro O., Michalick M.S.M., Costa R.T., Mayrink W. \& Oréfice F. 1999. Leishmaniose visceral canina: estudo parasitológico e histológico em olhos de cães. Parte I. Revta Bras. Oftalmol. 58(5):331-337. 
Diniz S.A., Melo M.S., Borges A.M., Bueno R., Reis B.P., Tafuri W.L., Nascimento E.F. \& Santos R.L. 2005. Genital lesions associated with visceral leishmaniasis and shedding of Leishmania sp. in the semen of naturally infected dogs. Vet. Pathol. 42:650-658.

Everrit B. 1974. Cluster analisys. Heinemann Education Books, London. $136 p$.

França-Silva J.C., Costa R.T., Siqueira A.M., Machado-Coelho G.L.L., Costa C.A., Mayrink W., Vieira E.P., Costa J., Genaro O. \& Nascimento E. 2003. Epidemiology of canine visceral leihmaniasis in the endemc area of Montes Claros Municipality, Minas Gerais state. Brazil. Vet. Parasitol. 111:161-173.

Galati E.A.B., Nunes V.L.B., Rego Jr F.A., Oshiro E.T. \& Chang M.R. 1997. Estudo de flebotomíneos (Díptera: Psychodidae) em foco de leishmaniose visceral no Mato Grosso do Sul, Brasil. Revta Saúde Pública 31(4):378-390.

Genaro O., Mayrink W., Michalick M.S.M., Dias M., Da Costa C.A. \& Melo M.N. 1988. Naturally occurring visceral leishmaniasis in dogs: Clinical aspects. Mem. Inst. Oswaldo Cruz 83:43.

Giunchetti R.C., Mayrink W., Genaro O., Carneiro C.M., Corrêa-Oliveira R., Martins-Filho O.A., Marques M.J., Tafuri W.L. \& Reis A.B. 2006. Relationship between canine visceral leishmaniosis and the Leishmania (Leishmania) chagasi burden in dermal inflammatory foci. J. Comp. Pathol. 135:100-107.

Giunchetti R.C., Martins-Filho O.A., Carneiro C.M., Mayrink W., Marques M.J., Tafuri W.L., Correa-Oliveira R. \& Reis A.B. 2008. Histopathology, parasite density and cell phenotypes of the popliteal lymph node in canine visceral leishmaniasis. Vet. Immunol. Immunopathol. 121:23-33.

Hedger M.P. 1997. Testicular leukocytes: What are they doing? Rev. Reprod. 2:38-47.

Hedger M.P. \& Hales D.B. 2006. Immunophysiology of the male reproductive tract, p.1195-1286. In: Jimmy D. Neill (Ed.), Knobil and Neill's Physiology of Reproduction. $3^{\text {rd }}$ ed. Elsevier, New York.

Keenan C.M., Hendricks L.D., Lightner L., Webster H.K. \& Johnson A.J. 1984. Visceral leishmaniasis in the German shepherd dog. Infection, clinical disease, and clinical pathology. Vet. Pathol. 21:7479.

Mancianti F., Gramiccia M., Gradoni L. \& Pieri S. 1988. Studies on canine control: Evolution of infection of different clinical forms of canine leishmaniasis following antimonial treatment. Trans. R. Soc. Trop. Med. Hyg. 82:566-567.

Marsella R. \& Gopegui R.R. 1998. Leishmaniasis: A re-emerging zoonoses. Int. J. Dermatol. 37:801-814.
Monteiro S.P., Lacerda M.M. \& Arias J.R. 1994. Controle da leishmaniose visceral no Brasil. Revta Soc. Bras. Med. Trop. 27:67-72.

Moreno J. \& Alvar J. 2002. Canine leishmaniasis: Epidemiological risk and the experimental model. Trends Parasitol. 18:399-405.

Owens S.D., Oakley D.A., Marryott K., Hatchett W., Walton R., Nolan T.J., Newton A., Steurer F., Schantz P. \& Giger U. 2001. Transmission of visceral leishmaniasis through blood transfusions from infected English foxhounds to anemic dogs. J. Am. Vet. Med. Assoc. 219:10761083.

Reis A. 2001. Avaliação de parâmetros laboratoriais e imunológicos de cães naturalmente infectados com Leishmania (Leishmania) chagasi, portadores de diferentes formas clínicas da infecção. Tese de Doutorado, Universidade Federal de Minas Gerais, Belo Horizonte. $176 p$.

Reis A.B., Martins-Filho O.A., Teixeira-Carvalho A., Carvalho M.G., Mayrink W., França-Silva J.C., Giunchetti R.C., Genaro O. \& Corrêa-Oliveira R. 2006. Parasite density and impaired biochemical/hematological status are associated with severe clinical aspects of canine visceral leishmaniasis. Res. Vet. Sci. 81:6875.

Reis A.B., Martins-Filho O.A., Teixeira-Carvalho A., Giunchetti R.C., Carneiro C.M., Mayrink W., Tafuri W.L. \& Corrêa-Oliveira R. 2009. Systemic and compartmentalized immune response in canine visceral leishmaniasis. Vet. Immunol. Immunopathol. 128:87-95.

Serre V. \& Robaire B. 2002. Interations of the immune system and the epididymis, p.219-231. In: Robaire B. \& Hinton B.T. (Eds), The Epididymis: From molecules to clinical practice. Ed. Kluwer Academic, New York.

Setchell B.P. \& Breed W.G. 2006. Anatomy, vasculature, and innervation of the male reproductive tract, p.771-808. In: Neill J.D. (Ed.), Knobil and Neill's Physiology of Reproduction. $3^{\text {rd }}$ ed. Elsevier, New York.

Silva F.L., Oliveira R.G., Silva T.M.A., Xavier M.N., Nascimento E.F. \& Santos R.L. 2009. Venereal transmission of canine visceral leishmaniasis. Vet. Parasitol. 160:55-59.

Tesh R.B. 1995. Control of zoonotic visceral leishmaniasis: Is it time to change strategies? Am. J. Trop. Med. Hyg. 52:287-92.

Tryphomas L., Zawdzka Z., Bernard M.A. \& Janzen E.A. 1977. Visceral leishmaniasis in a dog: Clinical hematological and pathological observations. Can. J. Comp. Med. 41:1-12.

WHO 2003. Leishmaniasis: Geographical distribution. World Health Organization. Disponível em <http://www.who.int/leishmaniasis/ leishmaniasis_maps/en/> Acesso em 20 jan. 2010. 